\title{
The STEM and Retrovirus Structure
}

\author{
Martha N. Simon, Beth Y. Lin, and Joseph S. Wall \\ Biology Dept., Brookhaven National Laboratory, Upton, NY 11973
}

Most of the physical measurements on the structure of retroviruses were made around twenty years ago. These large enveloped viruses contain an RNA genome. The internal structural protein, Gag, comprises most of the mass of the virion. The stoichiometry of Gag in virions was inferred from these early indirect measurements. Also, the mature whole viruses ae somewhat heterogeneous in size.

The unique capability of the STEM is that isolated, individual particles can be viewed and their masses measured directly. To re-examine the mass of the prototypic avian retrovirus, Rous sarcoma virus (RSV) in order to determine the number of Gag proteins in the virion, Vogt's lab purified two strains of RSV. One strain had a genome of only Gag, Pol, and Env. The other carried an additional gene which made the genome $20 \%$ larger (more like the "real" RSV which carries an additional src oncogene). The purified viruses were examined in the STEM and their masses measured. The STEM data indicated that the population of virions was not homogeneous. Although both sets of masses were variable, they were about the same forthe two viral strains. One hypothesis for the mass variability was that the size might be determined by the amount of RNA packaged in the virus. It seems unlikely that the quantity of packaged nucleic acid is responsible for the variability in mass[1].

In addition, the principles of retrovirus assembly are not well understood. An early stage in assembly is an immature capsid. Retrovirus-like particles (VLPs) can assemble spontaneously in vitro from purified Gag protein and nucleic acid. The morphology of the VLPs can resemble immature cores. By manipulating the fragments of Gag protein and nucleic acid inin vitro assembly systems, stages in this process can be analyzed. Mass measurements from the STEM are a quantitative way of assessing the variability of the assembled VLPs as well as determining the number of Gag proteins per VLP. Two recent projects have used the STEM to study virus like particles (VLPs) assembled in vitro.

Details of assembly are being investigated in Vogt's lab using the retrovirus, RSV. VLPs will assemble with only purified Gag protein (or a Gag protein fragment containing the NC domain) in vitro in the presence of nucleic acid. RNAs of various sizes, double-stranded DNA, and single-stranded DNA oligonucleotides larger than $20 \mathrm{nt}$, all promoted assembly. The ratio of protein to nucleic acid was the same for all the VLPs. Parallel preparations of RNA and DNAoligomer containing particles were examined in the STEM. Masses of the two sets of VLPS were measured and appeared indistinguishable but somewhat heterogeneous[2].

In vitro assembly of Mason-Pfizer monkey virus (M-PMV) immature capsids is being studied in Hunter's lab. To determine the mass and population diversity of capsids selected from the cytoplasm of baculovirus-infected cells, M-PMV Gag particles were purified from a series of sucrose gradients and examined in the STEM. Mass measurements (again) demonstrated the 
heterogeneity of the particles. This seems to indicate a variable number of Gag polyproteins within these particles also. [3].

These studies demonstrate the unique usefulness of the STEM in directly measuring the mass and heterogeneity of isolated particles without the constraint or necessity of a presupposed model. Conclusions, such as the difference in the number if Gag proteins per virion depending on the type of retrovirus, are credible. Although all these viruses appear to have some local symmetry (see Fig. 1 R), it is not necessary to have, say, an icosahedral model to obtain informative results. Additional experiments are underway at the STEM to elucidate these structures. [4]

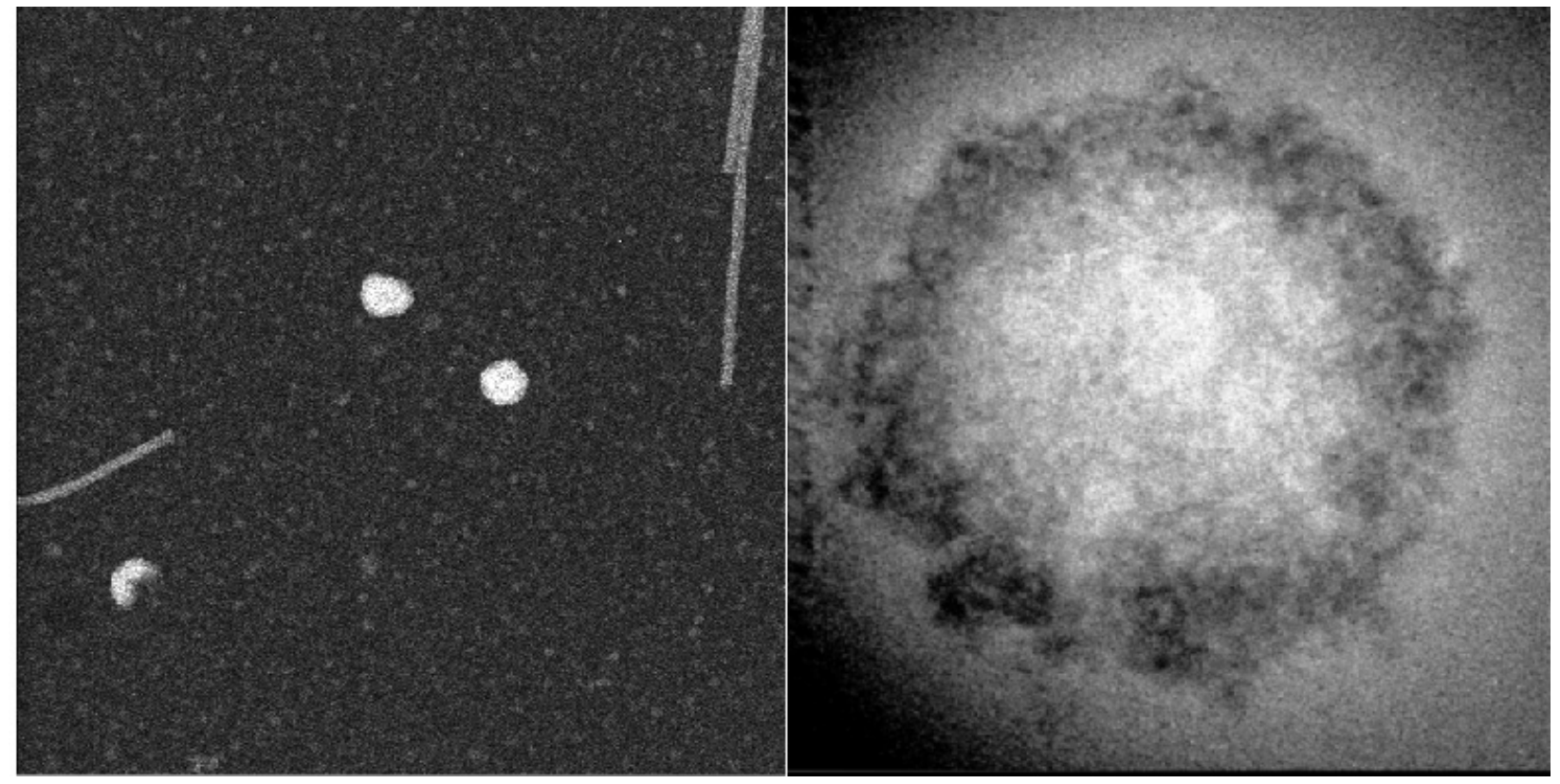

Fig. 1L(eft). Dark field STEM micrograph of 2 freeze-dried RSV-capsids (and 1 partial structure). The long rods are TMV (as a control). Full scale is $1.024 \mu \mathrm{m}$.

Fig. 1R(ight). Bright field STEM micrograph of a RSV capsid stained with methylamine vanadate (Nanovan). Full scale is $0.128 \mu \mathrm{m}$.

[1] Vogt, V. M. and Simon, M. N. (1999). Mass determination of Rous sarcoma virus virions by scanning transmission electron microscopy. J. Virol., 73, 7050-7055.

[2] Yu, F., Joshi, S. M., Ma, Y. M., Kingston, R. L., Simon, M. N., and Vogt, V. M. (2001). Characterization of Rous sarcoma virus Gag particles assembled in vitro. J. Virol. 75, 275364. [3] Parker, S. D., Wall, J. S. and Hunter, E. An analysis of Mason-Pfizer monkey virus Gag particles by scanning transmission electron microscopy. (2001) J Virol. 75: 95438.

[4] The BNL STEM is an NIH Supported Resource Center, NIH P41-RR01777, with additional support provided by DOE, OBER. 